\title{
The Recent Development of MIPO in Long Bone Fractures
}

\author{
Min Li", Xu Zhang", Xu Liü, Yang Jing \\ Tianjin Medical University, Tianjin, China. \\ Email: *812879698@qq.com \\ Received September $29^{\text {th }}, 2012$; revised October $29^{\text {th }}, 2012$; accepted November $16^{\text {th }}, 2012$
}

\begin{abstract}
Minimally invasive plate osteosynthesis (MIPO), which is developed under the guidance of biological osteosynthesis (BO) rules, can achieve faster and better rehabilitation. MIPO is mainly used in long bone fractures such as humerus, tibia and femur, but the technique is distinctive in each fracture site. The operative method, experimental outcome and comparison with other internal fixation methods will be discussed to determine whether MIPO is the best method or alternative method in fracture treatment. Other technique such as less invasive stabilizing system (LISS), which is developed on the basis of MIPO will also be introduced. In this review article, a general view of recent development MIPO is to be given.
\end{abstract}

Keywords: Minimally Invasive Plate Osteosynthesis; Biological Osteosynthesis; Less Invasive Stabilizing System

\section{Introduction of MIPO}

The Arbeitsgemeinschaft für Osteosythese (AO) technique is started in 1958 by M. E. Müller and his colleagues. They emphasized on precise reduction and fixation using mainly compression. Absolute stability of fixation achieved by using implants allowed the fractures to unite solidly [1]. What is more, an extensive surgery is usually required according to $\mathrm{AO}$ rules, consequently enhancing the risk of necrosis and delayed healing. However, precise reduction and absolute stable fixation has its biological price such as extensive soft tissue stripping and disruption of periosteal blood supply [2,3].

In order to deal with excessive pursuit of the stability of the fixed system mechanics, Gerbe [4] Palmar [5] have proposed a new concept of biological osteosynthesis (BO) since the early 90 s of last century. BO rules pay more attentions to the biological characteristics of the bone instead of destroying the normal physiological environment of bone growth and development. After that, the concept of biological internal fixation is rapidly developing. The basic idea of BO is, during fracture reduction and the process of fixation, maximized protection should be done to preserve the regional blood supply therefore healing of fractures becomes faster and prevent many complications.j

In recent years, Minimally invasive plate osteosynthesis (MIPO), a new technology developed under the guidance of $\mathrm{BO}$ rules is [6] has become widely accepted for treat-

\footnotetext{
*Corresponding author.

"The second and third authors contributed equally to this article.
}

ment of periarticular fractures, metaphyseal fractures, and certain diaphyseal fractures where intramedullary nailing (IMN) is not indicated [7]. There has been evidence showing the superiority of biological fixation over a stable mechanical fixation [8]. The use of MIPO prevents

1) Large surgical approach

2) Extensive soft tissue stripping

3) Disruption of periosteal blood supply

4) Nerve palsies

Moreover, MIPO shortened the union time after surgery and the period of rehabilitation. Thus, MIPO helps young people back to work as soon as possible and old people who are osteoporotic [9].

MIPO is now widely used in long bone fractures, mainly in humerus, tibia and femur. Operative procedures and characteristics are distinctive in different fracture sites. The passage below will show a general view of recent studies on MIPO.

\section{MIPO in Fractures of Humer}

\subsection{Fracture at Proximal Third Operative}

\subsubsection{Procedures}

Patients treated by MIPO technique firstly receive general anaesthesia with beach-chair position. A $5-\mathrm{cm}$ incision is made at the anteromedial border of the deltoid muscle, which is about $5-\mathrm{cm}$ distal to the acromium. Another $5-\mathrm{cm}$ incision is made at the lateral side of the distal shaft of the humerus. The distal incision is above the anatomical sign of the radial nerve, which is $8-\mathrm{cm}$ from the lateral epicondyle where the radial nerve pierces the 
lateral intermuscular septum and winds along the lateral border of the humerus. The muscle is split with blunt dissection forming a submuscular extraperiosteal tunnel, which lies between the brachial muscle and periosteum. The proximal fragment is usually positioned in an abducted and internally rotated position. In order to align the distal fragment in a reasonable alignment with the proximal fragment, a closed reduction is needed. The metaphyseal locking compression plate (LCP) is premoulded by image intensifier. The proximal fragment is fixed with three to four $3.5-\mathrm{mm}$ locking screws using bicortical fixation. The distal part can be fixed with two or more 5-mm locking screws also using bicortical fixation. A drain is unnecessary under most condition [10].

\subsubsection{Assessment and Discussions}

A study shows an average constant score was excellent among 17 patients who were treated by MIPO. Three of seventeen cases (17.6\%) suffered from neuropraxia. All except three patients $(82.4 \%)$ could achieve at least $140^{\circ}$ of shoulder abduction in the first 6 months after the operation. All except one (94.1\%) had bony union at 6 months [11].

Another study shows a mean radiological fracture union time of 12.9 weeks ranging from 10 to 20 weeks. The mean surgical time was 91.5 minutes and mean radiation exposure was 160.3 seconds. Shoulder function was excellent in 27 cases $(84.3 \%)$ and good in remaining 5 cases $(15.6 \%)$ on the UCLA score. Elbow function was excellent in 26 cases (81.2\%), good in 5 cases (15.6\%), and fair in 1 case (3.1\%) [12].

\subsection{Fracture at Mid and Distal Third}

\subsubsection{Operative Procedure}

A closed reduction is done under image guidance. A proximal and a distal incision to the fracture site are made on the anterior side of the arm respectively. A submuscular extraperiosteal tunnel is prepared between the brachial muscle and underlying periosteum by using elevators on both sides. Through this tunnel, a straight long narrow 4.5-mm wide dynamic compression plate (DCP) is inserted from the proximal incision, passing the fracture site and down to the distal incision. At least three screws should be inserted with bicortical fixation in each main fracture fragment. One of the screws is percutaneously inserted by an additional stab incision. The radial nerve is not exposed during the whole procedure and drain tube is not necessarily needed [10].

Fractures of the distal third of the humerus complicated by lesions of the radial nerve can also be treated by MIPO. Three incisions are made during operation. A 5.0 $\mathrm{cm}$ to $8.0 \mathrm{~cm}$ long oblique middle incision is made at the junction of the middle and distal thirds of the forearm. The radial nerve is exposed between the brachialis and brachioradialis muscles through this incision. Radial nerve is freed from its distal end to its emergence at lateral intermuscular septum, thus protected from injury. A proximal incision is made on anterior surface of humerus gaining proximal access between the biceps tendon medially and the tendon of deltoid laterally. A distal incision is made on lateral side of humerus gaining the distal access between tendon of brachioradialis and triceps tendon. A narrow DCP is inserted from the distal incision through middle incision to proximal incision. The arm is then abducted to between $60^{\circ}$ and $90^{\circ}$ to correct the varus deviation and the length was re-esta- blished by traction before attaching the plate. The proximal and distal fragments are then fixed with screws. The wound is closed without a drain or external fixation [13].

\subsubsection{Assessment and Discussions}

Many studies have reached a consensus that the humeral shaft fracture can be perfectly cured by MIPO [2].

The study which focusing on fractures of distal humerus with preoperative radial nerve palsy shows a satisfying result that all the fractures had healed at an average of 2.7 months, nerve lesion recovered at a mean of 2.3 months. Carrying angle of the elbow and a normal range of movement are re-established in all cases [13].

In fractures of mid and distal humerous without preoperative radial nerve lesion, the study reveals no significant differences existed in shoulder function assessed by UCLA score and elbow function assessed by MEPI score. Operation time between MIPO group and conventional group showed no significant discrepancy. However, MIPO group shows superiority over union time and occurrence of iatrogenic radial nerve palsy when compared to conventional group despite the suspicion of the safety of MIPO for material implant is so close to radial nerve in middle and distal humeral shaft [2,9].

Over all, MIPO contribute to low risk of iatrogenic radial nerve palsies and nonunion [10]. Most humer fractures treated by MIPO technique healed after about 12 15 weeks $[2,12,14]$.

\section{MIPO in Fractures of Tibia}

\subsection{Fracture at Distal Part}

\subsubsection{Operative Procedure}

MIPO technique can be operated on either medial or lateral side of tibia.

In medial MIPO, an anterior-medial curved incision about $3-6 \mathrm{~cm}$ is made exposing and protecting the saphenous vein. After closed reduction under image intensifier, the premoulded plate is inserted along the anteromedial surface and upward from the distal end proximally. Next, a proximal incision about three holes long is made to expose the upper part of the plate. When the 
plate position was adjusted as needed, three locking screws at each side are driven in [15].

In lateral MIPO, a closed reduction is also performed by manual traction under the support of $\mathrm{C}$-arm fluoroscopy to obtain alignment of the distal tibia. A $2-3 \mathrm{~cm}$ anterolateral incision is made at distal tibia extending proximally along the anterolateral surface of tibia. After a 3 - $4 \mathrm{~cm}$ proximal skin incision, subcutaneous tunnel is created by blunt dissection. A plate is inserted between the extensor hallucis longus tendon and the tibialis anterior tendon, or the extensor hallucis long tendon and the extensor digitorum longus tendon. The plate is inserted from the distal to proximal. Distal tibia should be fixed with a cancellous screw or three cortical screws, and the proximal tibia should be fixed with more than three cortical screws. Then, a joint spanning external fixator is removed $[16,17]$.

Fixation of fibula fractures used to be considered unnecessary. Nevertheless, when instability of the inferior tibiofibular syndesmosis is diagnosed, internal fixation of the fibula is necessary. Malleolar fractures, even with no significant displacement, should also be a matter of concern [15].

MIPO technique can be used in either open or closed fracture in fractures of distal tibia.

In open fracture, a 3-stage therapy is demanded. At stage 1 , all dead soft and bone tissues are removed by definitive wound lavage and debridement. Temporary ankle-spanning external fixator, either mono or delta frame, is performed within 24 hours. Delayed primary closure (DPC) as well as vaccum-assisted closure (VAC) is used. After the stage 1 treatment and a delay for a mean period of 15 days, the status of soft tissue is properly stabilized enough for soft tissue reconstruction. With no sign of infection, stage 2 surgical treatments are performed with MIPO technique after closed reduction. Stage 3 is not necessary for every patient. A bone graft is performed after MIPO to obtain bone union only in cases with a severe metaphyseal bone defect and lack of progression of healing for over 8 weeks in the distal tibia [16].

In closed fracture, LCP is inserted through submuscular extraperiosteal plain and then fixed to proximal and distal fragments after closed reduction is attempted [15].

\subsubsection{Assessment and Discussions}

It is demonstrated that wound complication of MIPO, such as wound infection and implant impingement, in distal tibia fractures are relatively common. [18] A study reveals that IMN should be the first method of treatment for the internal fixation of open tibia fractures [17]. Another study showed that MIPO group has no significant difference in healing time, time of recovery to work and ankle function assess by mazur score system when compared with ORIF group in closed fracture [15]. However, when the fracture line is less than $5 \mathrm{~cm}$ proximal to the ankle joint, IMN is not applicable [15]. MIPO technique can be an alternative method for those cases IMN is not available [17].

\subsection{Intra-Articular Fracture at Proximal Part}

\subsubsection{Operative Procedure}

Double osteosynthesis which is defined as a lateral MIPO combined with medial external fixator is needed at proximal fractures of tibia. A lateral MIPO is performed by two incisions on the lateral side of tibia after adjusted the alignment of distal part with proximal fragment. Medial unilateral frame with three pins, one in the proximal fragment and two in the distal fragment, span the whole tibia. After surgery, the leg is elevated with a posterior above-knee splint until soft tissue swelling resolved. Knee motion is started on the third day after operation. After a mean time of 8 weeks (range 6 - 10 weeks), the external fixator can be removed in patients with radiological evidence of progressive fracture consolidation [19].

LISS is a newly developed implant designed for fractures at proximal tibia and distal femur based on MIPO technique, functioning as "internal-external fixator". The anatomically pre-contoured plate made of titanium alloy has specially designed screws which thread and lock in the plate holes. All screws are placed using the irrigation system to cool while drilling. Bicortical screws are used in the metaphysis and monocortical screws used in the diaphysis. The anchorage of screw head in the plate allows the screws to be in a position forming a fixed angle with blade plate. The entire implant can be inserted using small incisions. The length of the plate is selected to obtain a minimum of three good screws distal to the fracture and it is better to obtain four screws if allowed. All LISS fixators are placed on the lateral side of the tibia, and pinned proximally and distally. LISS exert no compression on the interface between the plate and the bone, resulted in preservation of periosteal vessels [20, 21].

\subsubsection{Assessment and Discussions}

Lateral MIPO combined with a medial external fixator have been proved to be a fairly good method of fixation in terms of results and complications. [19] In a study of 22 patients, all fracture healed uneventfully. No wound dehiscence or infection was noted. At the final physical examination, the mean range of motion in all operated knees was flexion $132^{\circ}$ (range $115^{\circ}-150^{\circ}$ ) and extension $-3^{\circ}$ (range $-8^{\circ}$ to $+4^{\circ}$ ) [19].

LISS reduces soft tissue injury and damage of blood supply by avoiding compression on bones. In patients treated by LISS, a medial external fixator is also in need. LISS is weak in a varus direction owing to the lateral 
position, so it is valuable to support the eccentric load carrier temporarily with an external fixator on the medial side when treating unstable fractures [20]. Another outcome of patients with open fractures treated by LISS to measure was the incidence of deep and superficial infection. James P Stannard et al. described the rate of infection using the LISS implants in open fractures is not higher than the use of other contemporary methods of stabilization [21].

\section{MIPO in Fractures of Femur}

\subsection{Fracture at Distal Third}

\subsubsection{Operative Procedures}

Under fluoroscopic guidance, a transverse incision is made over the lateral aspect of the distal femur at the level of the intercondylar notch. The breadth of the incision is 0.5 to $1 \mathrm{~cm}$ more than the maximum width of the metaphyseal flare of the plate. The tensor fascia lata was split along the direction of its fibres. A submuscular tunnel is created by using an osteotome. Following reducetion, appropriate-sized plates (with 6 to 12 holes) are inserted in a distal-to-proximal direction over the lateral aspect of the distal femur. The length of the plate is determined intraoperatively. The proximal extent should be long enough to hold at least 3 screw holes and distal extent should not beyond the joint line. All proximal screws are bicortical. For distal fixation, at least 3 metaphyseal locking screws should be used and their sizes do not violate the intercondylar notch space. Another incision was made over the lateral aspect of the thigh at the level of the proximal screw holes. This proximal incision is used to check the approximation of plate to bone and fix the screws in. Reduction is sometimes maintained by Kirschner wires placed through holes in the LCP. Postoperatively, the operated limb is kept in elevation on a splint with the knee in $10^{\circ}$ to $15^{\circ}$ of flexion [22].

LISS is also used in the femoral fracture. In intra-articular fracture at distal femur, the patient is positioned supine on the radiolucent table with the knee flexed to avoid the typical hyperextension of the distal fragment caused by the pull of the gastrocnemius muscle. After painting and draping, a lateral skin incision aligned to Gerdy's tubercle is made at the distal femur. The iliotibial band is exposed and incised in line with the skin incision. After elevation of the vastus lateralis muscle, a premoulded LISS plate long enough to comply with the principle of bridging osteosynthesis is inserted under the vastus lateralis muscle proximally. Fracture reduction and implant positioning are verified by biplanar intraoperative fluoroscopic imaging system. The plate position was secured by K-wires. Five self-cutting, selftapping, fixed-angle screws are inserted into the fragments using trocar system after the removal of K-wires. All screws are tightened by using a dynamometric screwdriver and irrigated to cool the bone. Deep suction drain is placed and the wound is closed in layers [23].

\subsubsection{Assessment and Discussions}

Distal femoral fractures are associated with high-energy trauma (in the youngsters) and osteoporotic bones (in the elderly) [24]. The studies of MIPO in the treatment of distal femoral fractures have shown improved results over traditional methods. Ravi M Nayak et al. reported MIPO using a LCP achieves favorable biological fixation for distal femoral fractures with few complications. Bone grafting is not needed even in cases of metaphyseal comminution [22].

LISS is also an effective way for the treatment of distal femur fractures for it is useful in treating complex distal femoral fractures. LISS results in reduced blood loss, low infection rates and early mobility due to primary stability $[25,26]$. In a retrospective consecutive study, and 50 patients are treated by LISS. Deep wound infection was seen in one patient and mal-positioning with cutting-out of the proximal screws was seen in two patients. All other fractures healed uneventfully without bone graft requirements after a mean of 12 weeks [27]. Comparison between LISS and dynamic condylar screw (DCS) shows that LISS have lower risk of early implant loosening than the DCS [28]. However, the result of comparison between retrograde nailing and LISS showed no differences in outcome between implants regarding fracture healing, nonunion, and infection [23]. Therefore, LISS is a good alternative to conventional extramedullary or intramedullary stabilizing techniques, especially in more complex fracture situations [29].

\subsection{Fracture at Proximal and Mid Third}

\subsubsection{Operative Procedures}

The patient is placed in a supine position on the radiolucent operating table. Small $(4-5 \mathrm{~cm})$ proximal and distal incisions are made over the lateral aspect of the femur with deep dissection. Both two incisions go down through the ilio-tibial tract and vastus lateralis muscle in line with their fibres to the plane between the periosteum and the vastus lateralis muscle. The lateral cortex of the femur is exposed at both incisions by using two Hohmann retractors, one ventral and one dorsal on each incision site. A submuscular extraperiosteal tunnel is created from the proximal incision towards the distal incision by tunneling instrument. One end of the plate is tied to the hole at the tip of the tunneling instrument by means of a suture. The attached plate is pulled into the prepared tunnel while withdrawing the tunneling instrument. The image intensifier was used to check the correct position of the plate. Screw placement was done by two different techniques depending on surgeon preference. Three or four 
adjacent screws can be inserted through the proximal or distal incision while three or four separated screws require independent incisions for percutaneous screws to obtain more stable fixation [30].

\subsubsection{Assessment and Discussions}

Various treatment methods have been used for proximal femoral fractures [31]. There are several reports indicating that MIPO has also achieved satisfactory clinical outcomes and promoted the fracture healing in proximal fracture of femur [32].

For a femoral shaft fracture, closed IMN of the femur is still considered to be a standard treatment [32]. However, the results of femoral shaft fractures treated with MIPO are comparable to those treated with IMN. A study reported 10 cases of Vancouver type B1 femoral shaft fractures treated with percutaneous cerclage wiring and MIPO resulted in satisfactory reduction, adequate stability and healing in nine patients [33]. What is more, this technique can be used for femoral shaft fractures of all types [30]. Therefore, MIPO is a good choice for patients in whom IMN is contraindicated.

\section{Conclusions}

MIPO is a good choice in the treatments of long bone fractures in upper and lower extremities. It can be used either independently or along with external fixation, either in closed or open fracture with a 3-stage therapy. In humer, MIPO reduce the risk of iatrogenic radial nerve palsy and rate of nonunion. No significant differences were found in elbow and shoulder function. It is suggested that MIPO can be used as the first choice in fractures of humer. In tibia, wound complication is relatively common in MIPO group and MIPO group showed no superiority over ORIF group in healing time, time of recovery to work and ankle function. IMN is still the first choice while MIPO can work as an alternative method. However, MIPO combined with external fixator have been proved to be a fairly good method in terms of results and complications. In femur, MIPO has also achieved a satisfactory result and are comparable to IMN. MIPO can also be used in complicated fractures which IMN is not indicated. Thus, MIPO is suggested to be the first choice for fractures at femur although IMN is currently used as a standard treatment.

In fractures around knee joint, LISS is also an effective way for the treatments of fractures around knee which resulted in reduced blood loss, low infection rates, early mobility and lower risk of early implant loosening. Shorter and better recovery is achieved by avoiding compression on bone. In patients treated with LISS, a medial external fixator is suggested to support the eccentric load.

MIPO can only be used in certain fracture sites and fracture types at present. However, major limitations of
MIPO and LISS in clinical usage are high price and complexity of technological process.

Although MIPO technique is not widely used currently, it has become more popularized and well accepted during the last few years. As the development of surgical technology and material science, MIPO technique will be further progressed. MIPO is reported also having been used in radial, ulnar and clavicular fractures recently. In the future, MIPO will be able to be used in all kinds of fractures and LISS will be able to be used in joints other than knees. MIPO may take the place of traditional internal fixation method owing to its advantages of preservation of soft tissue and periosteal blood supply.

\section{REFERENCES}

[1] S. M. Perren, "Fracture Healing. The Evolution of Our Understanding," Acta Chirurgiae Orthopaedicae et Traumatologiae Cechoslovaca, Vol. 75, No. 4, 2008, pp. 241246.

[2] Z. An, B. Zeng, X. He, Q. Chen and S. Hu, "Plating Osteosynthesis of Mid-Distal Humeral Shaft Fractures: Minimally Invasive versus Conventional Open Reduction Technique," International Orthopaedics, Vol. 34, No. 1, 2010, pp. 131-135.

[3] M. Wagner and R. Frigg, "AO Manual of Fracture Management: Internal Fixators: Concepts and Cases Using LCP/LISS," TAC, 2006.

[4] C. Gerber, J. Mast and R. Ganz, "Biological Internal Fixation of Fractures," Archives of Orthopaedic and Trauma Surgery, Vol. 109, No. 6, 1990, pp. 295-303. doi:10.1007/BF00636165

[5] R. H. Palmer, "Biological Osteosynthesis," The Veterinary Clinics of North America Small Animal Practice, Vol. 29, No. 5, 1999, pp. 1171-1185.

[6] Krettek C: Foreword, "Concepts of Minimally Invasive Plate Osteosynthesis," Injury, Vol. 28 No. S1, 1997, pp. A1-A2. doi:10.1016/S0020-1383(97)90108-X

[7] T. Apivatthakakul, O. Arpornchayanon, S. Bavornratanavech, "Minimally Invasive Plate Osteosynthesis (MIPO) of the Humeral Shaft Fracture. Is It Possible? A Cadaveric Study and Preliminary Report," Injury, Vol. 36, No. 4, 2005, pp. 530-538. doi:10.1016/j.injury.2004.05.036

[8] F. Baumgaertel, M. Buhl and B. A. Rahn, "Fracture Healing in Biological Plate Osteosynthesis," Injury, Vol. 29, No. S3, 1998, pp. C3-C6. doi:10.1016/S0020-1383(98)95002-1

[9] B. Livani, W. Belangero, K. Andrade, G. Zuiani and R. Pratali, "Is MIPO in Humeral Shaft Fractures Really Safe? Postoperative Ultrasonographic Evaluation," International Orthopaedics, Vol. 33, No. 6, 2009, pp. 1719-1723. doi:10.1007/s00264-008-0616-X

[10] A. Zhiquan, Z. Bingfang, W. Yeming, Z. Chi and H. Peiyan, "Minimally Invasive Plating Osteosynthesis (MIPO) of Middle and Distal Third Humeral Shaft Fractures," Journal of Orthopaedic Trauma, Vol. 21, No. 9, 2007, pp. 628-633. doi:10.1097/BOT.0b013e31815928c2 
[11] T. W. Lau, F. Leung, C. F. Chan and S. P. Chow, "Minimally Invasive Plate Osteosynthesis in the Treatment of Proximal Humeral Fracture," International Orthopaedics, Vol. 31, No. 5, 2007, pp. 657-664. doi:10.1007/s00264-006-0242-4

[12] M. S. Shetty, M. A.Kumar, K. Sujay, A. R. Kini and K. G. Kanthi, "Minimally Invasive Plate Osteosynthesis for Humerus Diaphyseal Fractures," Indian Journal of Orthopaedics, Vol. 45, No. 6, 2011, pp. 520-526.

[13] B. Livani, W. D. Belangero and R. Castro de Medeiros, "Fractures of the Distal Third of the Humerus with Palsy of the Radial Nerve: Management Using Minimally-Invasive Percutaneous Plate Osteosynthesis," The Journal of Bone and Joint Surgery British Volume, Vol. 88, No. 12, 2006, pp. 1625-1628. doi:10.1302/0301-620X.88B12.17924

[14] J. M. Concha, A. Sandoval and P. N. Streubel, "Minimally Invasive Plate Osteosynthesis for Humeral Shaft Fractures: Are Results Reproducible?" International Orthopaedics, Vol. 34, No. 8, 2010, pp. 1297-1305. doi:10.1007/s00264-009-0893-Z

[15] Cheng W, Li Y, Manyi W, "Comparison Study of Two Surgical Options for Distal Tibia Fracture-Minimally Invasive Plate Osteosynthesis Vs. Open Reduction And Internal Fixation," International Orthopaedics, Vol. 35, No. 5, 2011, pp. 737-742. doi:10.1007/s00264-010-1052-2

[16] O. J. Sohn and D. H. Kang, "Staged Protocol in Treatment of Open Distal Tibia Fracture: Using Lateral MIPO," Clinics in Orthopedic Surgery, Vol. 3, No. 1, 2011, pp. 69-76.

[17] C. Kayali, H. Agus, A. Eren and S. Ozluk, "How Should Open Tibia Fractures Be Treated? A Retrospective Comparative Study between Intramedullary Nailing and Biologic Plating," Turkish Journal of Trauma \& Emergency Surgery, Vol. 15, No. 3, 2009, pp. 243-248.

[18] T. W. Lau, F. Leung, C. F. Chan and S. P. Chow, "Wound Complication of Minimally Invasive Plate Osteosynthesis in Distal Tibia Fractures," International Orthopaedics, Vol. 32, No. 5, 2008, pp. 697-703. doi:10.1007/s00264-007-0384-z

[19] S. Singh, P. R. Patel, A. K. Joshi, R. N. Naik, C. Nagaraj and S. Kumar, "Biological Approach to Treatment of Intra-Articular Proximal Tibial Fractures with Double Osteosynthesis," International Orthopaedics, Vol. 33, No. 1, 2009, pp. 271-274.

[20] C. A. Mueller, C. Eingartner, E. Schreitmueller, S. Rupp, J. Goldhahn, F. Schuler, K. Weise, U. Pfister and N. P. Suedkamp, "Primary Stability of Various Forms of Osteosynthesis in the Treatment of Fractures of the Proximal Tibia," The Journal of Bone and Joint Surgery British Volume, Vol. 87, No. 3, 2005, pp. 426-432. doi:10.1302/0301-620X.87B3.14353

[21] J. P. Stannard, C. G. Finkemeier, J. Lee and P. J. Kregor, "Utilization of the less-Invasive Stabilization System Internal Fixator for Open Fractures of the Proximal Tibia: A Multi-Center Evaluation," Indian Journal of Orthopaedics, Vol. 42, No. 4, 2008, pp. 426-430.

[22] R. M. Nayak, M. R. Koichade, A. N. Umre and M. V. Ingle, "Minimally Invasive Plate Osteosynthesis Using a
Locking Compression Plate for Distal Femoral Fractures," The Journal of Orthopaedic Surgery, Vol. 19, No. 2, 2011, pp. 185-190.

[23] C. Hierholzer, C. von Ruden, T. Potzel, A. Woltmann and V. Buhren, "Outcome Analysis of Retrograde Nailing and Less Invasive Stabilization System in Distal Femoral Fractures: A Retrospective Analysis," Indian Journal of Orthopaedics, Vol. 45, No. 3, 2011, pp. 243-250.

[24] K. A. Egol, E. N. Kubiak, E. Fulkerson, F. J. Kummer and K. J. Koval, "Biomechanics of Locked Plates and Screws," Journal of Orthopaedic Trauma, Vol. 18, No. 8, 2004, pp. 488-493. doi:10.1097/00005131-200409000-00003

[25] L. W. Xu, W. P. Ji, H. Li, P. F. Zhao and B. Wu, "Treatment of Osteoporotic Distal Femur Fractures with Less Invasive Stabilizing System (LISS) and High Strength Injectable Graft," China Journal of Orthopaedics and Traumatology, Vol. 23, No. 2, 2010, pp. 144-146.

[26] P. Kanabar, V. Kumar, P. J. Owen and N. Rushton, "Less Invasive Stabilisation System Plating for Distal Femoral Fractures," The Journal of Orthopaedic Surgery, Vol. 15, No. 3, 2007, pp. 299-302.

[27] W. Kolb, H. Guhlmann, C. Windisch, F. Marx, K. Kolb and H. Koller, "Fixation of Distal Femoral Fractures with the Less Invasive Stabilization System: A Minimally Invasive Treatment with Locked Fixed-Angle Screws," The Journal of Trauma, Vol. 65, No. 6, 2008, pp. 1425-1434. doi:10.1097/TA.0b013e318166d24a

[28] F. C. Kao, Y. K. Tu, J. Y. Su, K. Y. Hsu, C. H. Wu and M. C. Chou, "Treatment of Distal Femoral Fracture by Minimally Invasive Percutaneous Plate Osteosynthesis: Comparison between the Dynamic Condylar Screw and the Less Invasive Stabilization System," The Journal of Trauma, Vol. 67, No. 4, 2009, pp. 719-726. doi:10.1097/TA.0b013e31819d9cb2

[29] M. Schutz, M. Muller, P. Regazzoni, D. Hontzsch, C. Krettek, C. Van der Werken and N. Haas, "Use of the Less Invasive Stabilization System (LISS) in Patients with Distal Femoral (AO33) Fractures: A Prospective Multicenter Study," Archives of Orthopaedic and Trauma Surgery, Vol. 125, No. 2, 2005, pp. 102-108. doi:10.1007/s00402-004-0779-x

[30] T. Apivatthakakul and S. Chiewcharntanakit, "Minimally Invasive Plate Osteosynthesis (MIPO) in the Treatment of the Femoral Shaft Fracture where Intramedullary Nailing Is Not Indicated," International Orthopaedics, Vol. 33, No. 4, 2009, pp. 1119-1126. doi:10.1007/s00264-008-0603-2

[31] D. Hontzsch, "Distal Femoral Fracture-Technical Possibilities," Kongressband Deutsche Gesellschaft fur Chirurgie Kongress, Vol. 118, 2001, pp. 371-374.

[32] R. A. Winquist, S. T. Hansen, D. K. Clawson Jr., "Closed Intramedullary Nailing of Femoral Fractures. A Report of Five Hundred and Twenty Cases," The Journal of Bone and Joint Surgery American Volume, Vol. 66, No. 4, 1984, pp. 529-539.

[33] T. Apivatthakakul, C. Phornphutkul, T. Bunmaprasert, K. Sananpanich, A. Fernandez Dell'Oca, "Percutaneous Cerclage Wiring and Minimally Invasive Plate Osteosyn- 
thesis (MIPO): A Percutaneous Reduction Technique in the Treatment of Vancouver Type B1 Periprosthetic Femoral Shaft Fractures," Archives of Orthopaedic andTrau- ma Surgery, 132, No. 6, 2012, pp. 813-822. doi:10.1007/s00402-012-1489-4 